\title{
RoboCup Small-Size League: Past, Present and Future
}

\author{
Alfredo Weitzenfeld ${ }^{1(凶)}$, Joydeep Biswas ${ }^{2}$, Mehmet Akar ${ }^{3}$, \\ and Kanjanapan Sukvichai ${ }^{4}$ \\ ${ }^{1}$ University of South Florida, Tampa, FL, USA \\ aweitzenfeld@usf.edu \\ 2 Carnegie Mellon University, Pittsburgh, PA, USA \\ joydeepb@ri.cmu.edu \\ 3 Boğaziçi University, Istanbul, Turkey \\ mehmet.akar@boun.edu.tr \\ ${ }^{4}$ Kasetsart University, Bangkok, Thailand \\ sukvichai@gmail.com
}

\begin{abstract}
The Small Size Robot League (SSL) was among the founding RoboCup leagues in the 1997 competition held during IJCAI'97 in Nagoya, Japan. Since then, the league has experienced various advances in terms of robot design, number of robots, field size, software algorithms and other infrastructure used during the games, among these the recent standardization of the vision system shared by all teams. The SSL league has been one of the fastest paced leagues in RoboCup where teamwork, coordination, high-level strategies and artificial intelligence have played a critical role in the league development. As robots speeds have greatly increased in the past years, the league has witnessed the development of advanced control and cooperative algorithms. In parallel, shared open software, in particular the shared vision system has made it easier for new teams to join the league. In this paper we discuss the past, present and future of the Small Size League in its path towards the goal of achieving robot vs. human soccer in 2050.
\end{abstract}

Keywords: Small-size league - Shared vision - Omnidirectional control · Artificial intelligence $\cdot$ Wheeled robots

\section{Introduction}

Since the original robot soccer proposal by Mackworth [1] and the following RoboCup initiative [2], the Small Size League (SSL) has been a unique and pioneering league within the RoboCup initiative consisting of an off-board vision system to perceive all robots in the field [3]. The global vision system simplifies the task of robot localization and mapping problems, enabling teams to focus more on the software algorithms, hardware and control engineering.

Since its foundation a SSL soccer game takes place between two teams of five and just recently six - robots, where each robot must conform to the F180 dimension rule specifying that individual robots must fit within an $180 \mathrm{~mm}$ diameter circle and must be no higher than $15 \mathrm{~cm}$. An orange golf ball has been used since SSL foundation, with 
the robots playing soccer on a green carpeted field with its size having increased throughout the years [4], as shown in Fig. 1.

The complete game configuration is shown in Fig. 2. All objects on the field robots and ball - are tracked by a global vision system that processes the data provided by two cameras that are attached to a camera bar located $4 \mathrm{~m}$ above the playing surface. The shared vision system, i.e. SSL-Vision [5], is an open source project maintained by the league's community. The shared vision perceptions are processed by off-field computers belonging to each of the playing teams to provide wireless control of team robots, typically using a dedicated commercial FM transmitter/receiver unit. The offfield computers also receive communication from a referee box or game controller providing state of the game. Typically, these computers also perform most, if not all, of the processing required for coordination and control of the robots.

In general, building a successful team requires clever design, implementation and integration of many hardware and software sub-components into a robustly functioning whole making Small Size robot soccer a very interesting and challenging domain for research and education. In the rest of the paper we will briefly highlight the past, present and future of the league.

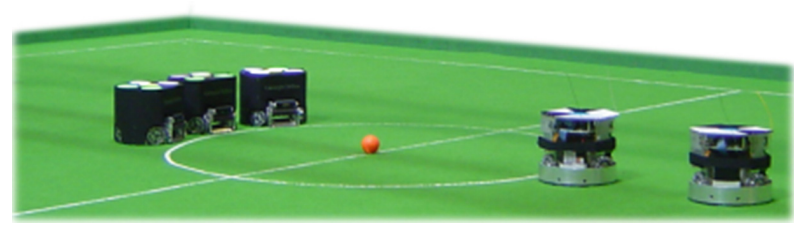

Fig. 1. Small size league robots facing against each other at the start of a game with orange golf ball at the center of green carpeted field (Color figure online).

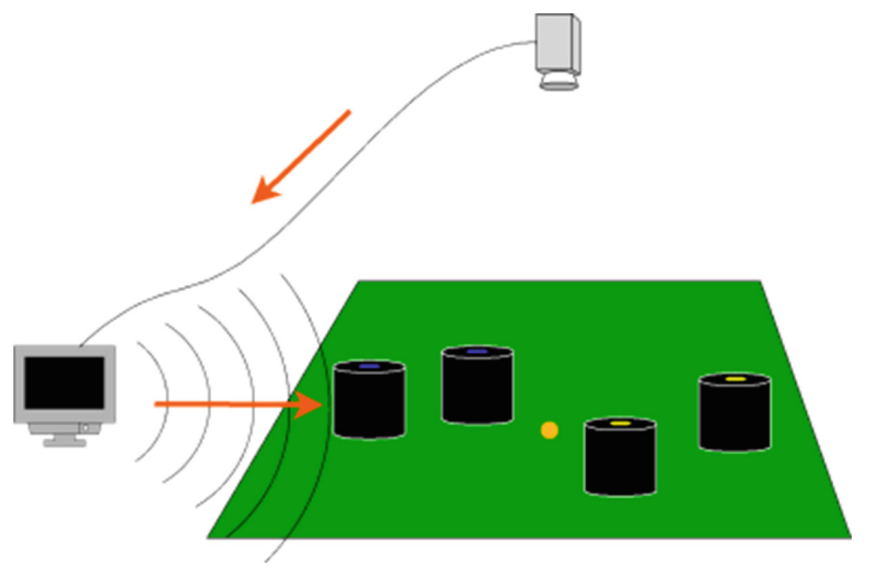

Fig. 2. Diagram illustrates the small size league robot control configuration with global vision system feeding off-field computer with ball position and robot positions and orientations. The offfield computer provides wireless control to the blue and yellow labeled team robots (Color figure online). 


\section{Past}

The Small Size Robot League (SSL) rules constraints have evolved over the years since 1997. The SSL robots in 1997 were limited to have a maximum linear dimension of $18 \mathrm{~cm}$ and a maximum projected area of $180 \mathrm{~cm}^{2}$. Today, the rules limit the robots to have a maximum diameter of $180 \mathrm{~mm}$ and maximum height of $150 \mathrm{~mm}$. In 1997, SSL teams were limited to a maximum of 5 robots, and this limit was only recently increased to 6 robots in 2012. The global vision system was initially processed through a single overhead camera, but this was later increased to two cameras, and with the introduction of the double-size field in 2014, to four cameras. The most important changes in time involved primarily the increase in field size and the robot design, starting as two wheeled differential control to the current four wheeled omnidirectional drive. Many of the initial challenges in SSL are still current as described by the CMUnited-97 team that won the first SSL competition [6].

\subsection{Vision}

One of the primary challenges of the SSL since 1997 had been to devise fast and accurate vision processing algorithms that could process camera images of the field an estimate the locations of all the robots and the ball on the field. The constraints of the vision system are numerous, and the vision system must:

1. Be capable of processing all the images in real time at full frame rates, which today consist of images of size $780 \times 580$ pixels captured at a rate of $60 \mathrm{~Hz}$,

2. Have minimal latency so as not to adversely affect the motion control of the robots,

3. Be capable of simultaneously tracking multiple robots and the ball on the field,

4. Be robust to robots touching each other without losing track of each individual robot, and

5. Correctly disambiguate between different robots in different orientations on the field, even when touching each other.

Due to these limitations, novel image processing algorithms, including CMVision [7] had to be developed to efficiently and robustly track all the robots and the ball by color segmentation of the images. CMVision has since been adopted as the de facto vision processing algorithm not only by the SSL, but also the other leagues of RoboCup, including the Standard Platform League.

\subsection{Robot Design}

The first soccer-playing robots in the SSL were differential-drive robots with two actuated wheels per robot and one or more passive castors. Since then, the drive systems of the SSL robots have evolved to use omnidirectional wheels, in particular the "Swedish Wheel" or "Meccanum Wheel" designs [9]. Initially three-wheel designs were most common among teams. However, the three-wheel designs suffered from significantly varying maximum acceleration and velocity profiles as a function of the 
directions that the robots drove along. To combat this problem, teams later adopted four-wheel designs, which provided more even distribution of the maximum acceleration and velocity as a function of the drive direction. The motors used in initial designs were brushed DC motors, whereas the SSL robots today use 3-phase brushless motors which are more efficient, provide higher torque, and more durable. New control strategies had to be developed in order to control the omnidirectional robots, including a real-time near-optimal minimum time motion controller, introduced by the SSL team Cornell Big Red [10].

SSL robots in 1997 had no special actuators to manipulate the ball, and instead bumped into the ball to kick it around the field. The first dedicated kicking mechanisms used rack-and-pinion linear actuators to propel the ball forward. Today, custom solenoid based kickers are used to efficiently and powerfully kick the ball at speeds of up to $15 \mathrm{~m} / \mathrm{s}$, although they are limited by software to comply with the kicking speed limits imposed by the rules. In addition to the main kicker, most teams have an additional solenoid kicker called the "chip-kicker" that can be used to propel the ball into the air to pass over opponent robots. A third manipulation mechanism involves a dribbling horizontal roller that is spun up at high speeds to impart back-spin to the ball on contact, thus allowing robots to hold on to the ball for short periods of time.

\section{Present}

There have been gradual changes to the Small-Size League rules since 1997 until now, among these, the addition of 1 robot for a total of 6 robots in each team, the introduction of a shared global vision system currently processing two overhead cameras, a significant increase in the field size, and most important the drastic advancement in the design of each robot from the original two wheels to the current four wheels. The ball is a standard orange golf ball, approximately $46 \mathrm{~g}$ in mass and $43 \mathrm{~mm}$ in diameter. In 2014 , the organizing committee is offering teams to compete in either the single-sized field of $6050 \mathrm{~mm} \times 4050 \mathrm{~mm}$ (see Fig. 3) or the optional double-size field of $8090 \mathrm{~mm} \times 6050 \mathrm{~mm}$. The double sized field will become the standard field for SSL in 2015. For either field, the playing surface is green felt mat or carpet and the floor under the carpet is level, flat, and hard. The field surface includes an additional $675 \mathrm{~mm}$ surface beyond the boundary lines on all sides. The outer $425 \mathrm{~mm}$ of this runoff area are used as a designated referee walking area. At the edge of the field surface, a $100 \mathrm{~mm}$ tall wall should prevent the ball and robots from running off the edge. All lines are $10 \mathrm{~mm}$ wide and painted white. The field of play is divided into two halves by a halfway line. The center mark is indicated at the midpoint of the halfway line. A circle with a diameter of $1000 \mathrm{~mm}$ is marked around it (Fig. 4).

A defense area is defined at each end of the field as follows for the two field sizes. In the single-size field, two quarter-circles of radius of $800 \mathrm{~mm}$ are drawn on the field of play. A line of length $350 \mathrm{~mm}$ parallel to the goal line connects these quarter circles. In the double-size field, two quarter-circles of radius of $1000 \mathrm{~mm}$ are drawn on the field of play. A line of length $500 \mathrm{~mm}$ parallel to the goal line connects these quarter-circles. The area bounded by this arc and the goal line is the defense area. 


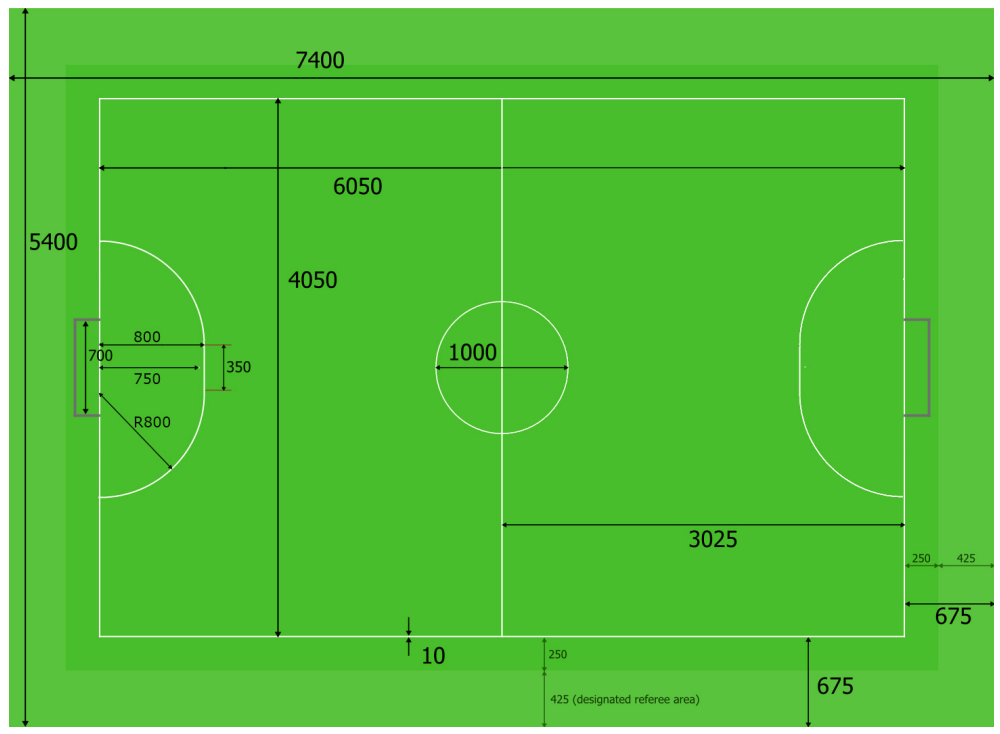

Fig. 3. The field dimensions of the single-size field. The dimensions include boundary lines. Dimensions of the field, goals, and special field areas are in millimeters.

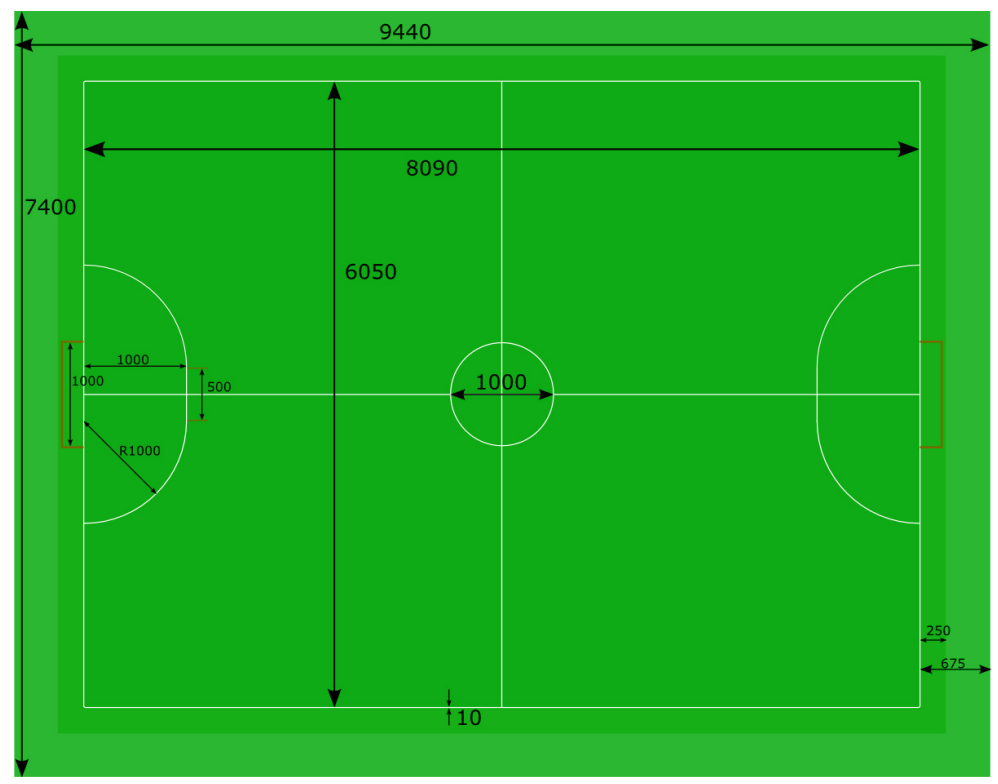

Fig. 4. The field dimensions of the single-size field. The dimensions include boundary lines. Dimensions of the field, goals, and special field areas are in millimeters. 
On the single-size field, within each defense area a penalty mark is made $750 \mathrm{~mm}$ from the midpoint between the goalposts and equidistant to them. On the double-size field, for each field half the penalty mark is $1000 \mathrm{~mm}$ from the midpoint between the goalposts and equidistant to them, thus coinciding with the outer edge of the defense area arc. The mark is a $10 \mathrm{~mm}$ diameter circle of white paint.

Goals are placed on the center of each goal boundary and anchored securely to the field surface. They consist of two $160 \mathrm{~mm}$ vertical side walls joined at the back by a $160 \mathrm{~mm}$ vertical rear wall. The inner face of the goal is covered with an energy absorbing material such as foam to help absorb ball impacts and lessen the speed of deflections. The goal walls, edges, and tops are white in color. There is a round steel cross bar that runs across the top of the goalmouth and parallel to the goal line. It is no thicker than $10 \mathrm{~mm}$ in diameter, but is sufficiently strong to deflect the ball. The bottom of the bar is $155 \mathrm{~mm}$ from the field surface, and the bar is dark in color to minimize interference with the vision system. The top of the goal is covered in a thin net to prevent the ball from entering the goal from above. It is attached securely to the cross bar and goal walls. The distance between the sidewalls is $700 \mathrm{~mm}$ for the single-size field and $1000 \mathrm{~mm}$ for the double-size field and the goal is $180 \mathrm{~mm}$ deep. The goal walls are $20 \mathrm{~mm}$ thick and touch the outer boundary of the field at the goal line, but do not overlap or encroach on the field lines or the field. The floor inside the goal is the same as the rest of the playing surface.

A game lasts two equal periods of $10 \mathrm{~min}$. Teams are entitled to an interval at half time. The half-time interval must not exceed $5 \mathrm{~min}$. Each team is allocated four timeouts at the beginning of the match. A total of $5 \mathrm{~min}$ is allowed for all timeouts. For example, a team may take three timeouts of one-minute duration and thereafter have only one timeout of up to two minutes duration. Timeouts may only be taken during a game stoppage. The time is monitored and recorded by the assistant referee who controls the a referee signaling device supplied during the game to convert the referee's commands into Ethernet communication signals that are transmitted to both teams. For games on the double-size field, the number of allocated timeouts is increased to six timeouts and the total time to $7.5 \mathrm{~min}$.

\subsection{Shared Vision System}

Each field is provided with a shared central vision server and a set of shared cameras. This shared vision equipment uses the community-maintained SSL-Vision software to provide localization data to teams via Ethernet in a packet format. Teams need to ensure that their systems are compatible with the shared vision system output and that their systems are able to handle the typical properties of real-world sensory data as provided by the shared vision system (including noise, latency, or occasional failed detections and misclassifications). Teams are not allowed to mount their own cameras or other external sensors, unless specifically announced or permitted by the respective competition organizers. The two or four cameras depending on whether a single-size or double-size field is used are mounted across bars provided $4 \mathrm{~m}$ above the field. The bar 
runs above the filed midline across goals in the case of the single-size field, while additional bars and cameras are added in the double-size field configuration.

Additionally, all teams must adhere to the operating requirements of the shared vision system having a flat surface on their top containing a unique "butterfly" color pattern [8] as shown in Fig. 5. The color of the robot top must be black or dark grey and have a matte (non-shiny) finish to reduce glare. Before a game, each of the two teams has a color assigned, namely yellow or blue. All teams must be able to be either yellow or blue color. The assigned team color is used as the center marker color for all of the team's robots. No two robots are allowed to use the same color assignment.

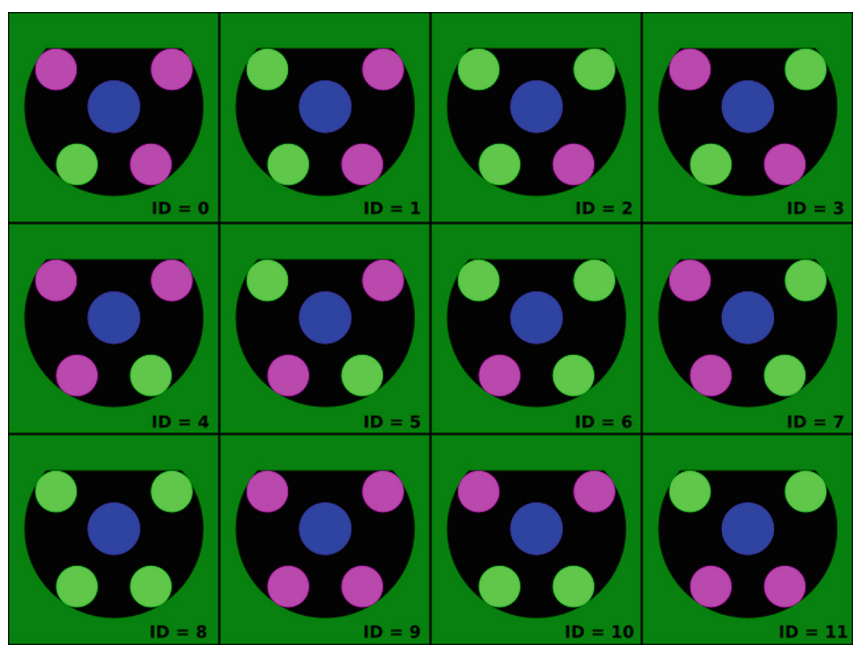

Fig. 5. The standard color assignments for use with the shared vision system.

\subsection{Robot Design}

A robot must fit inside a $180 \mathrm{~mm}$ diameter cylinder and have a height of $150 \mathrm{~mm}$ or less as shown in Fig. 6 (left). Robot wheels (or other surfaces that contact the playing surface) must be made of a material that does not harm the playing surface. Robots can use wireless communication to computers or networks located off the field. The robotic equipment is to be fully autonomous. Human operators are not permitted to enter any information into the equipment during a match, except at half time or during a time-out.

Dribbling devices may be included in the design of the robot as shown in Fig. 6 (right) as long as they actively exert backspin on the ball while the spin exerted on the ball must be perpendicular to the plane of the field. Vertical or partially vertical dribbling bars, also known as side dribblers, are not permitted. The use of dribbling devices has certain restrictions of usage during a game. 


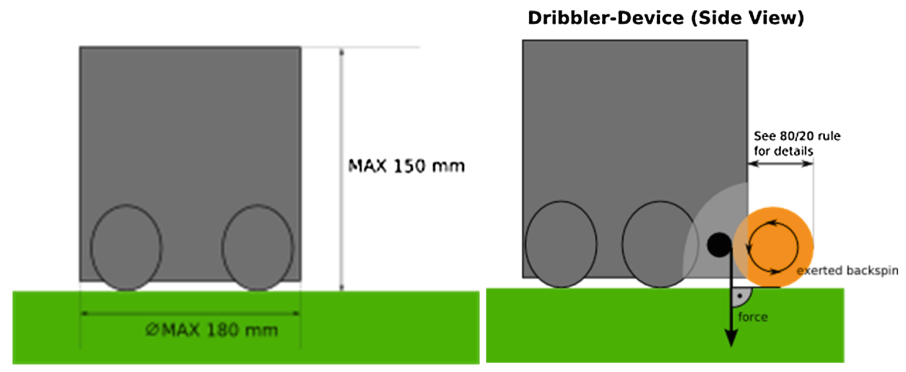

Fig. 6. (Left) The maximum robot dimensions; (Right) How a dribbler may work.

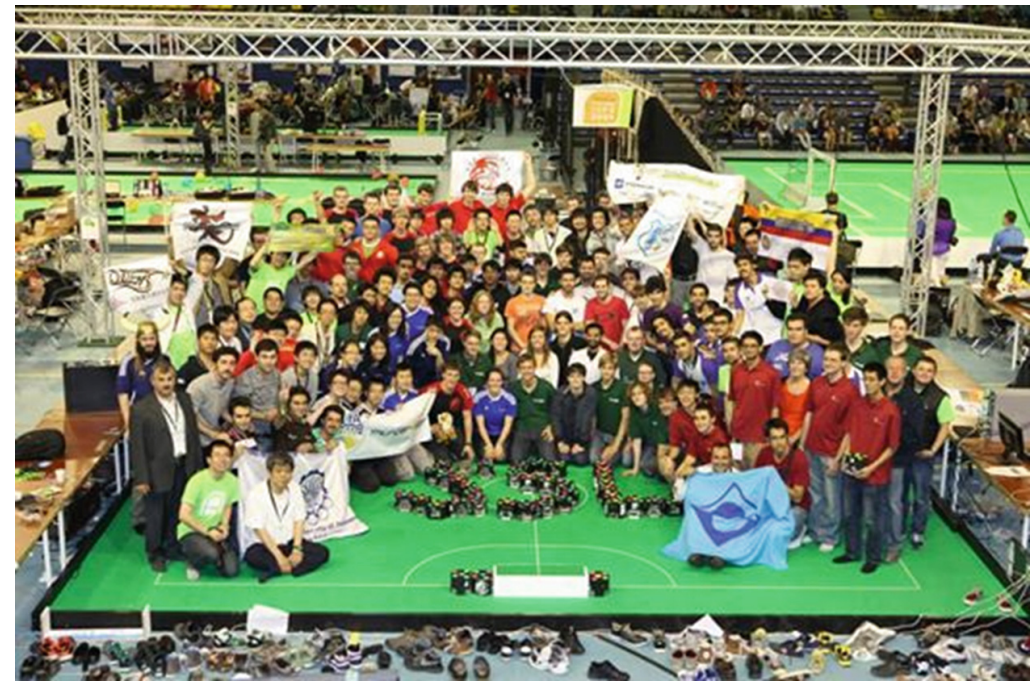

Fig. 7. Robocup small size league 2013 participants.

\subsection{Artificial Intelligence and Team Coordination}

One aspect that makes the Small Size League so attractive and unique among all RoboCup soccer leagues involving physical robots is the advanced pace of the game. The multi-robot aspect of soccer has been one of the most challenging areas of research in the SSL right from its inception. The first teams performed mostly as individual units of robots even on the same team, with no planned passes among teammates. The SkillsTactics-Plays (STP) architecture [11] was among the first formal approaches to plan for coordination among teammates, and is still used by most of the teams in the SSL. More recently, there has been an increasing interest in modeling opponents in order to learn their plays [12], robot ball passing and shooting [13], and dynamic selection, planning and learning of behaviors and strategies $[14,15]$. Robot deception, in the form of the "Coerce And Attack Planner" [16], was introduced in the SSL in RoboCup 2013 to 
coerce opponents away from strategic locations, allowing goals to be scored by exploiting the strategic openings.

\section{Future}

There are several aspects that the league has recently discussed as part of its future roadmap. We discuss immediate and future league changes. The small size league is planning to move by 2015 to the double-sized field. The main reason for the increase is having more space for robot game playing. The increase also includes a larger defense area and corresponding rule changes to reduce the number of penalty shots due to the defense trying to cope with the increasingly aggressive offense. An aspect that is currently being discussed is what should be the ratio of goal width to player size as it is currently far smaller than in real soccer. To make the goal area more realistic, we are considering length scales and rules more in line with the FIFA laws [17].

In addition to field increase goal width and defense area should be increased accordingly. In particular, the goal width should be increased such that even an entire team cannot block it. The goal width to player ratio of real soccer $(7.32 \mathrm{x})$ is appropriate for this, and this would lead to a goal width of $1.3 \mathrm{~m}$. There is also a need to amend the rules to balance offense and defense with the larger goal width and goal area. Note that this increase in field increase considers maintaining the same sized F180 robot as specified since the beginning of SSL.

\subsection{Shared Vision System}

The increase in field size implies technical challenges to the existing shared vision system and possibly its conversion to a totally different system. We are currently analyzing different alternatives that should be further evaluated in the future:

- Include additional field markings for calibration with 4 cameras with the existing SSL-Vision.

- Use tripod - mounted cameras with wide-angle and telephoto lenses to cover the field.

- Switch to a completely different vision system, like the Vicon motion capture system.

- Add a non-vision system such as RFID tags under the carpet or other radio based system.

\subsection{Robot Design}

At this moment we do not have any plans of changing the size of the robot in order to keep compatibility with existing robots and many of the existing league benefits such as easiness for transportation, inexpensive components, and established designs shared throughout the league. As other aspects of SSL are expected to change, such as field size, shared vision system, communication etc., we expect the robots to accommodate these aspects. 
Considering the great success of the shared vision system, we propose to include a shared communication system or "standard radio communications server" that all teams will use. This will avoid direct communication links from teams to their robots and would be comparable to other leagues using communication servers. A standardized communication server would also permit "pickup" games where robots from different teams may be exchanged and enable joint teams from different institutions.

An aspect directly related to the robot designs is the ball specification. Considering the need to restrict the kicking strength to limit ball speeds, we would like to consider aspects such as ball bounce and dynamics as compared to human soccer. Alternatives to the current golf ball include hockey and squash balls.

Given the increased capabilities of microcontrollers and the proposed inclusion of the standardized radio server, we will like to evaluate fully onboard computing without a PC controlling the robots. Instead, the PC would be used only to monitor the game and make offline changes to the robots.

\subsection{Artificial Intelligence and Team Coordination}

In terms of the advanced game playing, there are several proposals that have been discussed. Among these, the most important is the inclusion of an automatic refereeing system that is currently being tested. Other changes affect primarily the game itself including rules being applied.

We would like to discourage "unintelligent" gameplay by modifying rules involving far away kicks that directly score goals, such as chip kicks that travel more than half the width of the field, analogous to the "icing" rule in ice hockey.

We would like to increase the penalization for robot collisions, where for example, if two robots from opposing teams collide, then the team with the slower team gets an indirect free kick, and after 3 such indirect free kicks, the opposing team will get a yellow card. Implementing this rule will require an automated referee, and a formal definition of "collision" that the referee should be able to detect with a low false positive rate.

There is a need also to keep updating the game rules such as to balance the defense and offense, abolish the rule prohibiting multiple defenders in the defense area, and add the offside rule. This change might need additional checks like limiting the number of defenders in the defense area to the number of offense players plus one.

In terms of technical challenges, we would like to introduce compulsory technical challenges for teams, for example to proceed past the quarter finals, by making technical challenges more relevant to the game and geared to address technical shortcomings in the league, such as:

- Intercepting chip kicks (from both teammates as well as opponents).

- Obstacle avoidance with moving opponents.

- Score goals against opponents with fixed (but unknown) handicaps to promote AI, opponent modeling, learning, etc.

- Successfully steal a ball from an opponent robot.

- Advanced plays involving collaboration among multiple robots. 
Finally, we would like to evolve the league to have further interaction with humans as in real soccer games by introducing the role of the coach, though voice or written commands, so teams may modify their strategies during the game,

\section{Timeline}

We propose the following timeline:

- 2015: Play under double-size field.

- 2016: Introduction of standardized radio communication server.

- 2017: Introduction of automatic referee system and increase in number of robots (mixed teams).

- 2018: Introduction of fully onboard robot computing (elimination of controlling PC).

- 2019: Increase in field size to enable $11 \times 11$ robots and extension of shared vision system.

- 2020: Introduction of automated coaching during game.

\section{Conclusions and Discussion}

We have summarized in this paper the past, present and future of the small size league as currently envisioned by the executive committee based on prior discussion with the technical committee and team leaders. We summarized immediate challenges such as the recent increase to double the field size with corresponding changes such as the support of up to 4 cameras that will be tested in 2014 as a technical challenge and will become official in 2015. A critical aspect we would like to keep in the league is the size of the robots. As technology advances and component prices are reduced, in particular in relation to brushless motors, robots have become much more powerful while costing less. We would like to avoid a robot "power race" and instead see an advance in the "power algorithms" to control and coordinate among the robots to take advantage of the increased robot power by producing improved game play and collaborative agent strategies. We would also like to further encourage sharing of designs among teams, such as the introduction a few years back of mandatory ETDPs (Extended Team Description Papers) for top teams, and increase the use of shared systems in the league, such as the shared vision system (SSL-) that has encouraged new teams to enter the league. Finally, we would like to encourage Junior teams to take advantage of the advances of this league by developing a lower cost using established robotic kits or by exploiting lower cost components to develop a RoboCup Junior Small Size League analogous to that proposed several years ago [18].

Acknowledgements. The RoboCup Small Size League Executive Committee acknowledges the contribution of the members of the current and prior technical committees in specifying the rules of the league, the organizing committees in bringing these rules into actual competitions, the previous exec committees in guiding this league throughout the years, and the RCF trustees for 
supporting the SSL league. We also acknowledge all teams that have participated in SSL in the past years. Figure 7 shows a picture of RoboCup SSL 2013 participants.

\section{References}

1. Mackworth, A.K.: On seeing robots. In: Basu, A., Li, X. (eds.) Computer Vision: Systems, Theory, and Applications, pp. 1-13. World Scientific Press, Singapore (1993)

2. Kitano, H., Tambe, M., Stone, P., Veloso, M., Coradeschi, S., Osawa, E., Matsubara, H., Noda, I., Asada, M.: The RoboCup synthetic agent challenge 97. In: Proceedings of the Fifteenth International Joint Conference on Artificial Intelligence. Morgan Kaufman, San Francisco (1997)

3. Sahota, M.K., Mackworth, A.K., Barman, R.A., Kingdon, S.J.: Real-time control of soccerplaying robots using off-board vision: the dynamite testbed. In: IEEE International Conference on Systems, Man, and Cybernetics, pp. 3690-3663 (1995)

4. Small Size Robot League Wiki (2014). http://robocupssl.cpe.ku.ac.th/

5. Zickler, S., Laue, T., Birbach, O., Wongphati, M., Veloso, M.: SSL-vision: the shared vision system for the RoboCup small size league. In: Baltes, J., Lagoudakis, M.G., Naruse, T., Ghidary, S.S. (eds.) RoboCup 2009. LNCS, vol. 5949, pp. 425-436. Springer, Heidelberg (2010). http://code.google.com/p/ssl-vision/

6. Veloso, M., Stone, P., Han, K., Achim, S.: The CMUnited-97 small robot team. In: Kitano, H. (ed.) RoboCup 1997. LNCS, vol. 1395. Springer, Heidelberg (1998)

7. Bruce, J., Balch, T., Veloso, M.: Fast and inexpensive color image segmentation for interactive robots. In: Proceeding of the Intelligent Robots and Systems, pp. 2061-2066 (2000)

8. Bruce, J., Veloso, M.: Fast and accurate vision-based pattern detection and identification. In: Proceedings of ICRA2003: 2003 IEEE International Conference on Robotics and Automation, Taipei, Taiwan, pp. 1277-1282, May 2003

9. Diegel, O., Badve, A., Bright, G., Potgieter, J., Tlale, S.: Improved mecanum wheel design for omni-directional robots. In: Proceedings of the 2002 Australasian Conference on Robotics and Automation, Auckland, pp. 117-121, November 2002

10. Kalmár-Nagy, T., D’Andrea, R., Ganguly, P.: Near-optimal dynamic trajectory generation and control of an omnidirectional vehicle. Robot. Auton. Syst. 46(1), 47-64 (2004)

11. Browning, B., Bruce, J., Bowling, M., Veloso, M.: STP: skills, tactics, and plays for multirobot control in adversarial environments. J. Syst. Control Eng. 219(1), 33-52 (2005)

12. Erdogan, C., Veloso, M.: Action selection via learning behavior patterns in multi-robot domains. In: Proceedings of the International Joint Conference on Artificial Intelligence, pp. 192-197 (2011)

13. Nakanishi, R., Bruce, J., Murakami, K., Naruse, T., Veloso, M.M.: Cooperative 3-robot passing and shooting in the RoboCup small size league. In: Lakemeyer, G., Sklar, E., Sorrenti, D.G., Takahashi, T. (eds.) RoboCup 2006: Robot Soccer World Cup X. LNCS (LNAI), vol. 4434, pp. 418-425. Springer, Heidelberg (2007)

14. Trevizan, F.W., Veloso, M.M.: Learning opponent's strategies in the RoboCup small size league. In: 9th International Conference on Autonomous Agents and Multi-Agent Systems, Springer (2010)

15. Yasui, K., Kobayashi, K., Murakami, K., Naruse, T.: Analyzing and learning an opponent's strategies in the RoboCup small size league. In: Behnke, S., Veloso, M., Visser, A., Xiong, R. (eds.) RoboCup 2013. LNCS, vol. 8371, pp. 159-170. Springer, Heidelberg (2014) 
16. Biswas, J., Mendoza, J.P., Zhu, D., Choi, B., Klee, S., Veloso, M.: Opponent-driven planning and execution for pass, attack, and defense in a multi-robot soccer team. In: Proceeding of Autonomous Agents and Multi-Agent Systems, pp. 493-500 (2014)

17. FIFA, Laws of the Game (2013/2014) http://www.fifa.com/mm/document/footballdevelopment/ refereeing/81/42/36/log2013en_neutral.pdf

18. Baltes, J., Sklar, E., Anderon, J.: Teaching with RoboCup. In: Greenwald, L., Dodds, Z., Howard, A., Tejada, S., Weinberg, J. (eds.) Accessible Hands-on AI and Robotics Education. AAAI Spring Symposium Series, vol. 1, pp. 146-152 (2004) 